\title{
ENDOMETRITE SUBCLÍNICA APÓS O TRATAMENTO DE VACAS COM ENDOMETRITE CLÍNICA
}

\author{
Ana Rita Ferreira Moura1', Suzana Akemi Tsuruta1', Patrícia Magalhães Oliveira', \\ Nayara Resende Nasciutti ${ }^{1}$, Ricarda Maria Santos ${ }^{1}$, João Paulo Elsen Saut ${ }^{1}$ \\ 1 UFU \\ Correspondência: João Paulo Elsen Saut: jpsaut@famev.ufu.br
}

\begin{abstract}
RESUMO: A endometrite subclínica tem efeito negativo no desempenho reprodutivo pois reduz a taxa de prenhez e aumenta o intervalo parto-concepção. Diante disto, objetivou-se avaliar a ocorrência de endometrite subclínica (ES) aos 42 dias pós-parto (dpp) em vacas que foram diagnosticadas com endometrite clínica entre 21 e $28 \mathrm{dpp}$ e tratadas com infusão uterina de oxitetraciclina. Foram utilizadas 54 vacas mestiças leiteiras, divididas em dois grupos: o controle GC $(n=34)$, composto por vacas com parto e puerpério fisiológico de acordo com o exame clínico proposto e sem nenhum tratamento; e o grupo tratado - GT ( $n=20)$, vacas com endometrite clínica tratadas entre 21 e $28 \mathrm{dpp}$, com infusão uterina (4 g de oxitetraciclina) entre 21 e $28 \mathrm{dpp}$. Acompanhou-se no dia do parto, 7, 14, 21, 28 e 42 dpp. Realizou-se avaliação dos parâmetros vitais, escore de condição corporal, exame ginecológico e citologia endometrial através da técnica de cytobrush. Aos 42 dpp não houve diferença na porcentagem de muco vaginal limpo $(P=0,69)$ e os dois grupos apresentaram a mesma evolução clínica. Aos 42 dpp houve diferença entre a ocorrência de ES $(P<0.001)$ entre GC $(14,8 \%)$ e o GT $(80,0 \%)$, além de apresentar diferença na proporção de neutrófilos $(3,3 \pm 6,6 ; 54,6 \pm 39,9-p<0,001)$, células endometriais $(95,9 \pm 8,0 ; 39,6 \pm 40-p<0,001)$, macrófagos $(0,1 \pm 0,3 ; 1,1 \pm 1,0-p=0,016)$, linfócitos $(0,6 \pm 1,9 ; 3,9 \pm 4,2-p<0,001)$ e eosinófilos $(0,1 \pm 0,2 ; 0,8 \pm 2,0-p=0,113)$, respectivamente. Concluiu-se que, apesar das vacas com endometrite clínica apresentarem resposta ao tratamento com funções vitais, involução uterina e secreção vaginal semelhante às vacas com puerpério fisiológico, ainda persistem com uma alta incidência de endometrite subclínica (80\%) que representa um efeito negativo no desempenho reprodutivo.
\end{abstract}

Palavras-chave: bovinos; citologia endometrial; cytobrush; inflamação; útero

\section{SUBCLINICAL ENDOMETRITIS AFTER TREATMENT IN COWS WITH CLINICAL ENDOMETRITIS}

\begin{abstract}
Subclinical endometritis causes negative effects on reproductive performance how decreased conception rates and prolonged days open. The objective of this study was to evaluate the occurrence of subclinical endometritis (SE) at 42 days in milk (DIM) in cows that were diagnosed with clinical endometritis between 21 and 28 DIM and treated with uterine infusion of oxytetracycline. 54 crossbreed dairy cows were divided into two groups: GC $(n=34)$, animals that demonstrated physiological parturition and puerperium, based on the proposed clinical evaluation and without being treated; GT $(\mathrm{n}=20)$, cows with endometritis received uterine infusion (4 $\mathrm{g}$ of oxytetracycline) between 21 and 28 DIM. Cows were examined at parturition and at days 7, 14, 21, 28, and 42 DIM. The following procedures were performed: vital parameters and body condition score evaluation; gynecological exams and endometrial cytology using the cytobrush technique. There was no difference $(P=0.69)$ in the percentage of clear vaginal mucus between groups at 42 DIM, that demonstrated the same clinical evolution. At $42 \mathrm{DIM}$, there was significant difference $(P<0.001)$ in the occurrence of SE between GC (14.8\%) and GT (80\%) cows; and, endometrial cytology demonstrated significant differences in all cells that were evaluated between GC and GT cows: neutrophils $(39.9 \pm$ 6.6; $54.6 \pm 3.3-p<0.001)$; endometrial epithelial cells $(95.9 \pm 8.0 ; 39.6 \pm 40-p<0.001)$; macrophages $(0.1 \pm 0.3 ; 1.1 \pm 1.0-p=0.016)$; lymphocytes $(0.6 \pm 1.9 ; 3.9 \pm 4.2-p<0.001)$; and eosinophils $(0.1 \pm 0.2 ; 0.8 \pm 2.0-p=0.113)$, respectively. Although cows with clinical endometritis demonstrated response to treatment with clinical parameters, uterine involution and vaginal discharge similar to cows with physiological postpartum, there was an elevated incidence of subclinical endometritis $(80 \%)$ which has a negative effect on reproductive performance..
\end{abstract}

Key Words: bovine; endometrial cytology; cytobrush; inflammation; uterus 


\section{INTRODUÇÃO}

A abordagem fundamental para o sucesso na criação de gado leiteiro é a gestão do período periparto. Aspectos sobre a saúde uterina, a retomada da ciclicidade ovariana após a parição, assim como condições de nutrição e ambiente estão no foco deste interesse (Plöntzke et al., 2011).

Define-se por puerpério o período compreendido entre o parto e a completa involução uterina, aproximadamente, 40 dias pós-parto. Após a parição quatro acontecimentos devem ocorrer de forma concomitante: involução uterina, regeneração do endométrio, retorno à ciclicidade ovariana e eliminação da contaminação bacteriana (Sheldon, 2004). A infecção bacteriana uterina e os produtos bacterianos associados à inflamação podem suprimir a secreção do hormônio luteinizante (LH) pela pituitária, perturbando no pós-parto o crescimento folicular ovariano e posterior ovulação (Peter et al.,1989; Opsomer et al., 2000; Sheldon et al., 2002).

Nessa fase, a identificação precoce do animal doente e a adoção de cuidados para promover a sua recuperação o mais rápido possível são primordiais para minimizar as consequências da ocorrência das infecções uterinas e outras doenças puerperais concomitantes (Smith e Risco, 2005).

Os métodos mais comuns de diagnóstico a campo são através da palpação retal, vaginoscopia, ultrassonografia (Lewis, 1997) e a avaliação da presença de pus no muco vaginal; já que a severidade destas infecções pode ser verificada pelo exame das características e odor do conteúdo vaginal (Leblanc et al., 2002; Sheldon et al., 2002; Williams et al., 2005).

A endometrite clínica pode ser definida pela presença de descarga uterina purulenta na vagina detectável a partir do dia 21 após o parto, ou descarga uterina mucopurulenta presente na vagina a partir dos 26 dias após o parto (Sheldon et al., 2009).

Há uma série de terapias para a endometrite clínica, incluindo antibioticoterapia sistêmica ou local (Leblanc, 2008). O uso de infusões uterinas justifica-se por permitir altas concentrações no local da infecção, sendo utilizados vários princípios como a tetraciclina, penicilina, cefapirina, cloranfenicol, gentamicina, sulfonamida, oxitetraciclina, entre outras (Sheldon e Dobson, 2004; Leblanc, 2008). A oxitetraciclina é um antibiótico de amplo espectro, indicado para o tratamento e controle de infecções uterinas causadas por agentes Gram-positivos e Gramnegativos, podendo ser utilizada por infusão uterina (Azawi, 2008).

A técnica de infusão uterina é muito utilizada para o tratamento de endometrite, porém, ainda há muita dúvida sobre sua eficiência e possíveis efeitos deletérios à vida reprodutiva futura da vaca. Não há na literatura informação referente à incidência de endometrite subclínica em vacas tratadas com infusão uterina, apesar de a endometrite subclínica ser o foco de muitas pesquisas neste momento.

A endometrite subclínica tem um efeito negativo sobre o desempenho reprodutivo, pois reduz a taxa de prenhez e aumenta o intervalo partoconcepção (Kasimanickam et al., 2004; Gilbert et al., 2005; Bacha e Regassa, 2010; McDougall et al., 2010; Cheong et al., 2011), aumenta a incidência de vacas repetidoras de cio (Salasel et al., 2010) e a incidência de mastite subclínica (Bacha e Regassa, 2010). Diante do exposto, objetivou-se avaliar a ocorrência de endometrite subclínica após o tratamento de vacas com endometrite clínica. 


\section{MATERIAL E MÉTODOS}

Estudo conduzido na Fazenda Experimental do Glória da Universidade Federal de Uberlândia - UFU, município de Uberlândia, sudoeste do Estado de Minas Gerais, Brasil. O clima local é classificado como Aw (Köppen, 1948), com temperatura média anual de $22,3^{\circ} \mathrm{C}$, umidade relativa do ar em torno de $71 \%$ e precipitação pluviométrica de $1500 \mathrm{~mm}$ anuais.

Os animais foram mantidos sob sistema de pastejo no período das águas e em confinamento na seca, alimentados com silagem e durante a ordenha recebiam a suplementação de $1 \mathrm{~kg}$ de concentrado com $24 \%$ de proteína bruta para cada $3 \mathrm{~kg}$ de leite produzido.

Logo após o parto foram colhidas informações referentes à retenção de placenta, distocia, auxílio ao parto e distúrbios metabólicos. Os animais eram vacinados anualmente contra febre aftosa, raiva, clostridioses e leptospirose e realizado exame periódico para tuberculose, brucelose e leptospirose.

No pré-parto as vacas foram separadas individualmente até 0 momento do parto e depois transferidas para o lote lactação recebendo $1 \mathrm{Kg}$ de concentrado, $24 \%$ de proteína bruta, para cada $3 \mathrm{Kg}$ de leite produzido no momento da ordenha. A ordenha era realizada duas vezes ao dia.

As vacas mestiças, Holandês $x$ Gir, com composição genética variando de $1 / 2$ a $7 / 8$, foram divididas em dois grupos: grupo controle (GC), composto por 34 vacas que não apresentaram distocia e liberaram a placenta até 12 horas pós-parto, além disso, não apresentaram nenhuma alteração clínica de acordo com o exame clínico proposto (Feitosa, 2008) e não receberam tratamento no período avaliado; grupo tratamento (GT), composto por 10 vacas que apresentaram presença de secreção vaginal purulenta detectável aos $21 \mathrm{dpp}$ e 10 vacas com secreção vaginal mucopurulenta aos 28 dpp, diagnosticadas com endometrite clínica de acordo com Sheldon et al. (2009).

As vacas diagnosticadas com endometrite clínica $(n=20)$ foram tratadas, imediatamente, com infusão uterina de $20 \mathrm{ml}$ de oxitetraciclina diidrato diluída em solução fisiológica, correspondente a $4 \mathrm{~g}$ de oxitetraciclina base.

O exame clínico (Feitosa, 2008) foi realizado nos dias: parto (d0), 7 (d7), 14 (d14), 21 (d21), 28 (d28) e 42 (d42) dpp, quando foram avaliados os seguintes parâmetros: escore de condição corporal (ECC), classificando-as de 1 a 5 , sendo 1 considerada caquética e 5 obesa (Edmonson, 1989); parâmetros vitais: temperatura retal $\left(\mathrm{T}^{\circ} \mathrm{C}\right)$, frequência cardíaca $(F C)$, frequência respiratória $(F R)$, frequência ruminal (FRum) e coloração de mucosas.

No exame ginecológico, realizouse a palpação retal, exame ultrassonográfico, com o aparelho de ultrassom (DP-2200VET ${ }^{\circledR}$, Mindray, Shenzhen, China) equipado com transdutor retal linear de $5-7,5 \mathrm{MHz}$, no qual foram avaliados: diâmetro de corpo uterino, aferido imediatamente após a cérvix e cornos uterinos.

Finalmente, avaliou-se a secreção vaginal conforme a técnica descrita por Williams et al. (2005), com prévia limpeza da vulva com papel toalha, em seguida, utilizando luva de palpação, introduzia-se a mão na vagina, para remover o conteúdo. A classificação foi feita em relação à coloração e proporção de conteúdos: (1) secreção limpa e translúcida; (2) secreção sanguinolenta; secreção achocolatada; (4) secreção limpa com flocos de pus; (5) secreção mucopurulenta, conteúdo com $<50 \%$ de pus; (6) secreção purulenta, conteúdo com $>50 \%$ de pus. O muco também foi 
avaliado em relação ao odor, classificado como inodoro e fétido.

As amostras de citologia endometrial (CE) foram colhidas pela técnica de escova endometrial (cytobrush) (Kaufmann et al., 2009). A escova cervical não estéril (Labor Import $^{\circledR}$ ) foi adaptada e inserida em um aplicador universal de sêmen para a passagem através da cérvix. A vulva foi higienizada previamente com papeltoalha.

Após a passagem pela cérvix, chegou-se até a base do corpo uterino, onde o tubo foi retraído expondo a escova, que entrou em contato com o endométrio, foi rotacionada em sentido horário, então removida e rolada em lâmina previamente identificada, a qual foi transportada para o laboratório da Universidade Federal de Uberlândia para coloração pelo método de MayGrünwald Giemsa (Vallada, 1999). Depois das lâminas secas, foram submetidas à avaliação microscópica.

A análise da CE foi realizada pela avaliação do percentual de polimorfonucleares, a partir da contagem de 200 células, no aumento de 100X, sendo a leitura feita por dois observadores e considerando endometrite subclínica quando contados acima de $10 \%$ de neutrófilos.

A análise estatística foi feita utilizando-se os programas estatísticos Minitab Release 15 (Minitab Inc., Pensylvania, EUA) e Biostat 5.0 (Ayres et al., 2007); e a estatística descritiva apresentada em média, desvio-padrão e porcentagem. Para comparar parâmetros vitais, diâmetro uterino entre os grupos, as variáveis foram submetidas ao teste de KolmogorovSmirnov para avaliar a normalidade e, após, o teste $\mathrm{T}$ não-pareado (amostras paramétricas) ou o Teste Mann-Whitney (amostras não paramétricas). Para avaliar os momentos dentro dos grupos para CE utilizou-se o Teste de KruskalWallis com pós-teste de Dunn. O teste de exato de Fisher foi usado para comparar a frequência de endometrite subclínica, características e odor do muco vaginal. Todos os testes com níveis de significância igual a $5 \%$ (Vieira, 2003).

\section{RESULTADOS E DISCUSSÃO}

Em relação aos parâmetros vitais (FC, FR, Frum e coloração de mucosa palpebral), não houve diferença entre os grupos (Tabela 1) em todos os dias avaliados, além de permanecerem dentro dos valores de referência para a espécie (Feitosa, 2008) e para bovinos mestiços leiteiros criados em Minas Gerais (Saut et al., 2010).

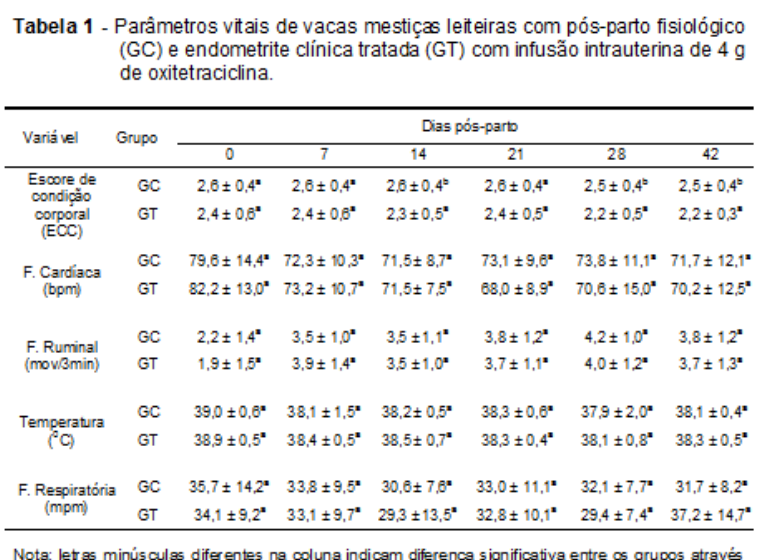

Nota: letras minúsculas diferentes na coluna indicam diferença significativa entre os grupos através Nota: letras minús culas diferentes
do Teste Mann-Whitney $(P=0,05$

A endometrite clínica é definida por uma inflamação superficial do endométrio sem comprometimento sistêmico (Sheldon, 2004; LeBlanc, 2008). Pela presença de descarga uterina purulenta na vagina detectável a partir de $21 \mathrm{dpp}$, ou descarga uterina mucopurulenta presente na vagina a partir dos 26 dpp (Sheldon et al., 2009), o que concorda com a classificação definida nesta pesquisa.

LeBlanc et al. (2002) ressaltaram que animais identificados com endometrite clínica, apresentando descarga uterina mucopurulenta, tendem a uma crescente queda na fertilidade pós-parto, esta situação 
torna-se pior quando são diagnosticados com descarga purulenta.

Os animais com endometrite clínica apresentaram ECC significativamente menor nos 14, 28 e 42 dpp. O ECC baixo na parição representa um impacto negativo na saúde pós-parto, lactação e reprodução (Hayirli e Grummer, 2004) e as vacas são mais propensas a ter um período de anestro prolongado quando apresentam ECC menor que 2,5 (Roche, 2006). Overton e Waldron (2004) sugeriram que vacas devem parir com escore corporal entre 2,75 e 3,00 e não perder mais que 0,5 pontos entre o parto e 0 primeiro serviço, contrariando recomendações anteriores (Buckley et al., 2003) de ECC mais elevados de 3,0 a 3,5.

Em relação à involução uterina, não houve diferença no diâmetro do corpo uterino (Tabela 2), avaliado por ultrassonografia transretal, e localização do útero na palpação retal. O diâmetro do corpo uterino variou entre $27,0 \pm 5,2$ a $19,4 \pm 4,5 \mathrm{~mm}$ e $25,5 \pm 5,2$ a $19,6 \pm$ $4,4 \mathrm{~mm}$ para o GT e GC, respectivamente.

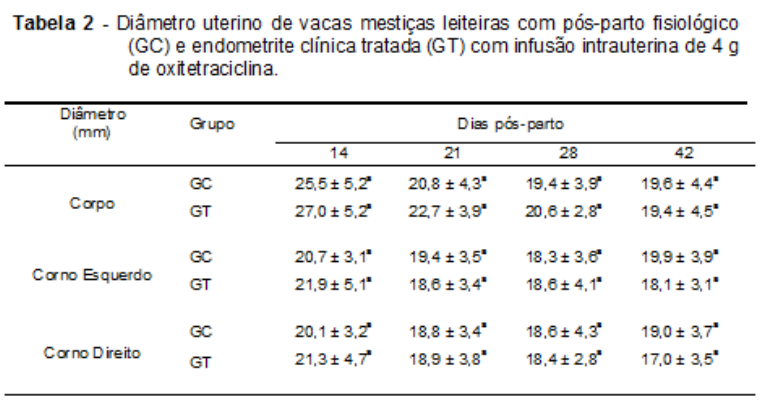

Nota: letras minúsculas diferentes
do Teste Mann-Whitney $(P=0,05$ )

LeBLanc et al. (2002) citaram que a endometrite em vacas Holandesas pode ser caracterizada por diâmetro cervical maior que $7,5 \mathrm{~cm}$ após $20 \mathrm{dpp}$; o presente estudo optou pela mensuração do corpo uterino, pois se observa uma variação muito grande do diâmetro cervical em vacas mestiças e os autores acreditam que não seja uma variável confiável na avaliação da presença de infecções uterinas nestes animais.

Sheldon (2004) mostrou o progresso da involução uterina através do diâmetro do corno anteriormente gravídico e do não gravídico. Ressaltou que 0 corno uterino anteriormente gravídico apresentava involução mais lenta, era mais largo e comprido na comparação com o corno uterino não gravídico, facilmente identificado até 28 dpp. No presente estudo não foi diferenciado corno gravídico de corno não gravídico. O diâmetro do corno esquerdo variou entre $21,9 \pm 5,1$ a 17,1 $\pm 3,1 \mathrm{~mm}$ para o GT e 20,7 $\pm 3,1$ a 19,9 $\pm 3,9 \mathrm{~mm}$ para GC e o corno direito variou entre $21,3 \pm 4,7$ a $17,0 \pm 3,5 \mathrm{~mm}$ de diâmetro para o GT e 20,1 $\pm 3,2$ a 19,0 \pm 3,7 mm de diâmetro para GC.

$\mathrm{Na}$ palpação retal as vacas do GC apresentaram o útero na cavidade abdominal, transição e pélvica, respectivamente, no d7 (78\%, 11\%, $11 \%)$, d 14 (27\%, 31\%, 42\%), d21 (11\%, $15 \%, 74 \%)$ e d $28(0 \%, 10 \%, 90 \%)$ e d 42 com $100 \%$ na cavidade pélvica. As vacas do GT apresentaram no d7 (83\%, $6 \%, 11 \%)$, d14 (30\%, 35\%, 35\%), d21 (5\%, 20\%, 75\%) e d28 e d42 com $100 \%$ do útero na cavidade pélvica à palpação retal. Não houve diferença significativa entre os grupos, em relação à porcentagem do útero na cavidade pélvica, talvez pela eficácia do tratamento utilizado no GT ou devido à palpação retal ser um método menos sensível para diagnóstico de endometrite clínica (LeBlanc et al., 2002).

Os métodos mais comuns de diagnóstico de endometrite clínica a campo são através da palpação retal, vaginoscopia, ultrassonografia (Lewis, 1997) e a avaliação da presença de pus na secreção vaginal, já que a severidade destas infecções pode ser verificada pelo exame das características e odor do conteúdo vaginal (Williams et al., 2005; LeBlanc et 
al., 2002; Sheldon, et al., 2002). A vaginoscopia pode predizer infecções uterinas em $59-82 \%$ dos casos, e apresenta correlação com a presença de Arcanobacterium pyogenes (Williams et al., 2005).

Consequentemente a incidência de endometrite pode ser afetada e distinta na dependência do método de diagnóstico utilizado, devido à insensibilidade e falta de especificidade da técnica de palpação retal e observação da secreção vaginal (Gilbert et al., 2005; Sheldon et al., 2006).

Houve diferença entre os grupos em relação ao odor em 14 ( $P=0,0042)$, $21(\mathrm{P}<0,0001)$ e $28 \quad(\mathrm{P}=0,0026) \mathrm{dpp}$, sendo que vacas do GT apresentaram odor fétido maior que as vacas do GC. Aos $42 \mathrm{dpp}$ a secreção vaginal não apresentou diferença em relação ao odor $(P=0,29)$ e características do muco $(P=0,69)$ entre os grupos $G C$ e GT (Figura 1). A frequência de muco limpo e translúcido foi de $85,2 \%$ e $76,5 \%$ para GC e GT, respectivamente, sugerindo que a intervenção do tratamento interferiu positivamente nas secreções vaginais (Figura 1).

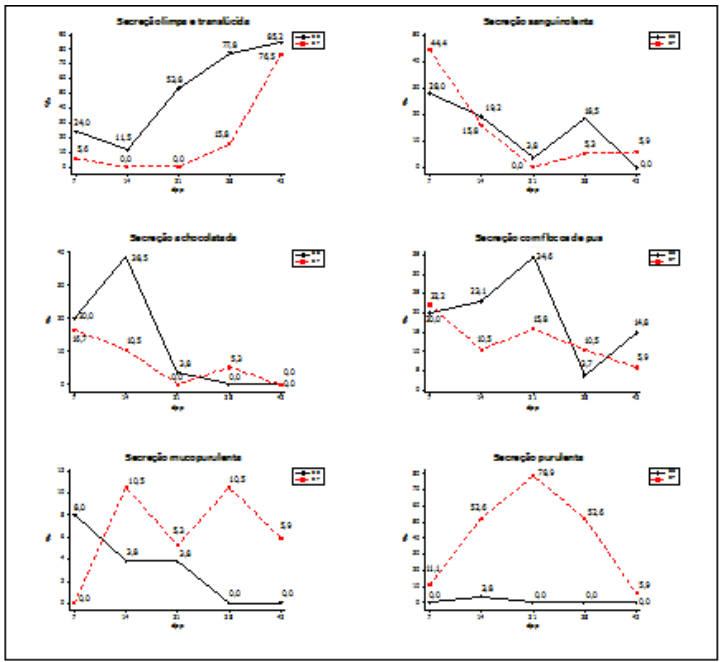

Figura 1 - Comparação da porcentagem (\%) dos tipos de secreção vaginal em relação (GC) oxitetracidina.

$\mathrm{Na}$ CE houve diferença em todas as células avaliadas no $42^{\circ} \mathrm{dpp}$ (Tabela $3)$, entre GT e GC: neutrófilos ( $p<0,001)$, células endometriais $\quad(p<0,001)$, macrófagos $\quad(p=0,016)$, linfócitos $(p<0,001)$ e eosinófilos $(p=0,113)$. Estudos recentes, consideram apenas 0 percentual de neutrófilos (Kasimanickam et al., 2004; Gilbert et al., 2005; Kasimanickam et al., 2005; Barlund et al., 2008; Bacha e Regassa, 2010; McDougall et al., 2010) para diagnóstico de ES, e não são avaliadas as outras células inflamatórias.

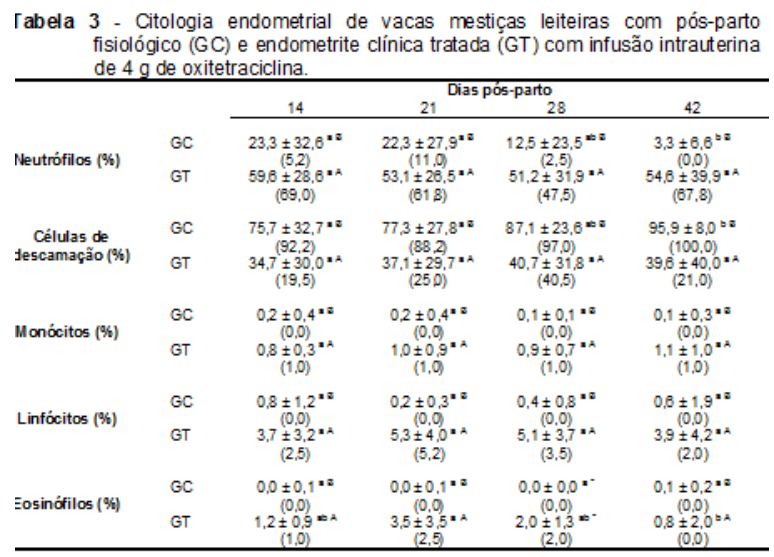

Vota: letras minúsculas diferentes na linha indicam diferença significativa entre os dpp nos grupos.

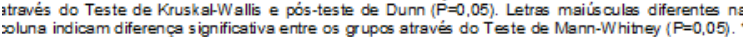
istatística não realizada devido acs valores do desvio padrăo igual a zero do GC

Houve diminuição gradativa na porcentagem de neutrófilos no pós-parto fisiológico (GC) de 23,3 \pm 32,6 (14ํำ dpp) para $3,3 \pm 6,6$ (42 $\mathrm{dpp}$ ). Segundo Sheldon et al. (2002), a principal barreira fagocítica é composta por neutrófilos que migram para o lúmen uterino em resposta à infecção uterina, apesar de saber que a capacidade funcional dos neutrófilos está reduzida neste momento (Baggiolini, 1998; Sheldon, 2004).

No pós-parto, a infecção uterina, causada por microorganismos do meio, pele e fezes (Sheldon 2004; Sheldon e Dobson 2004), diminui gradativamente nas primeiras semanas pós-parto (Grunert et al., 2005), o que justificaria a redução no número de neutrófilos. Conforme Elliot et al. (1968), algumas vacas podem permanecer infectadas e por este motivo permaneceria 0 estímulo, e permanência por mais tempo destas células inflamatórias, o 
que foi verificado no GT. Todas as vacas do GT permaneceram com aumento de todas as células inflamatórias aos 42 dpp, mesmo não existindo mais diferença na involução uterina e características da secreção vaginal comparado às vacas $\mathrm{GC}$.

Além do estímulo inflamatório provocado pela presença do agente infeccioso, as células inflamatórias aumentadas poderiam ser devido à persistência da inflamação sem 0 agente infeccioso ou à irritação provocada no endométrio pela oxitetraciclina e/ou o veículo utilizado na infusão uterina.

Em macacos e ratos as tetraciclinas provocaram lesões na transição entre útero e região intramural da tuba uterina, incluindo inflamação, cicatrização, necrose, fibrose e em alguns casos oclusão tubária (Dubin et al., 1984a; Dubin et al., 1984b; Zaneveld e Goldsmith, 1984). Gilbert e Schwark (1992), Smith e Risco (2005), Azawi (2008) e Leblanc (2008) citaram que a oxitetraciclina poderia causar lesões no trato reprodutivo, porém nenhum destes trabalhos tinha este objetivo, sendo apenas uma constatação relatada na discussão. Apesar de que, ShamsEsfandabadi et al. (2004), usando infusão de $1 \mathrm{~g}$ de oxitetraciclina $(20 \mathrm{ml}$ de oxitetraciclina $5 \%$ ) adicionados a $30 \mathrm{ml}$ de solução salina, não verificaram influência à primeira IA em vacas tratadas quando comparadas ao grupo controle.

Porém, independente do fator inicial, a presença de células inflamatórias já seria fator prejudicial ao desempenho reprodutivo. Além disso, a avaliação da porcentagem de polimorfonucleares apresentou valor superior ao exame bacteriológico e avaliação de secreção vaginal para predizer o desempenho reprodutivo (McDougall et al., 2010).

Marques Júnior et al. (2011) afirmaram a necessidade em se tratar os animais diagnosticados com endometrite clínica, já que nos casos mais graves, não são capazes de combater a infecção uterina, e os danos acarretados ao endométrio poderiam diminuir a capacidade de se tornarem gestantes (Sheldon, 2007; Gautam et al., 2010). Santos et al. (2010) demonstraram que a bactéria Arcanobacterium pyogenes, considerada a principal bactéria envolvida em metrites no pós-parto, apresentou alta resistência $(54,2 \%)$ para a oxitetraciclina, porém menor quando comparado a outros princípios como amoxicilina (56,9\%), ampicilina $(86,1 \%)$, cloranfenicol (100\%), florfenicol (59,7\%) e penicilina $(86,1 \%)$, apenas a tetraciclina apresentou valor inferior de $50 \%$.

A prevalência de endometrite subclínica (Tabela 4) em vacas com puerpério fisiológico diminuiu no decorrer do puerpério, concordando com os resultados descritos por Kasimanickam et al. (2004), Gilbert et al. (2005) e Plontzke et al. (2010). No GT não houve diminuição de endometrite subclínica esperada no decorrer do puerpério e houve diferença na porcentagem entre os grupos aos 28 e $42 \mathrm{dpp}$.

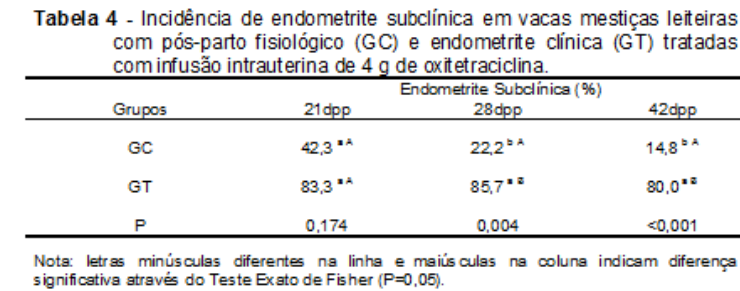

Kasimanickam et al. (2004), ao excluírem vacas com secreção vaginal purulenta, observaram incidência de endometrite subclínica de 35\% (20-33 dpp) em vacas Holandesas. Outras pesquisas não excluíram vacas Holandesas com secreções purulentas e encontraram incidência de endometrite subclínica de 11,1\% entre 28-41 dpp (Barlund et al., 2008), 53\% entre 40-60 
dpp (Gilbert et al., 2005), 38\% entre 1838 dpp (Plöntzke et al., 2010) e 25,9\% entre 40-60 dpp (Cheong et al., 2011).

Bacha e Regassa (2009), trabalhando com vacas cruzadas sem exclusão de animais com problemas pós-parto, identificaram incidência de $47,5 \%$ (28 dpp) e 30,5\% (56 dpp). Independente do delineamento experimental utilizado, a incidência de endometrite subclínica encontrada no grupo tratado com infusão intrauterina, aos $42 \mathrm{dpp}$, foi superior a todos os experimentos encontrados na literatura consultada. Esta alta incidência de endometrite subclínica, muito provavelmente, implicará em baixo desempenho reprodutivo nessa fase pós-parto (Kasimanickam et al., 2004; Gilbert et al., 2005; Bacha e Regassa, 2010; McDougall et al., 2010; Salasel et al., 2010; Cheong et al., 2011).

Ressalta-se que as técnicas de CE, os limites utilizados e os dias de avaliação no pós-parto não foram os mesmos entre as pesquisas descritas acima e, por este motivo, não podem ser avaliadas de maneira precisa. Estudos adicionais devem ser realizados, estabelecendo critérios adequados para interpretação da endometrite subclínica, verificando os fatores que levaram à elevada incidência da mesma nas vacas tratadas com infusão intrauterina de oxitetraciclina, avaliação da CE mais tardia no puerpério e, finalmente, comparar outros tratamentos de endometrite clínica em relação à incidência de endometrite subclínica no final do puerpério.

\section{CONCLUSÃO}

Vacas com endometrite clínica, diagnosticadas e tratadas com infusão intrauterina de $4 \mathrm{~g}$ de oxitetraciclina, entre 21 e 28 dias pós-parto, apresentam incidência de $80 \%$ de endometrite subclínica.

\section{AGRADECIMENTOS}

Os autores agradecem à Fundação de Amparo à Pesquisa do Estado de Minas Gerais - FAPEMIG, pelo apoio financeiro; Conselho Nacional de Desenvolvimento Científico e Tecnológico - CNPq, pela concessão de bolsa mestrado; e, Universidade Federal de Uberlândia, por ceder o local e rebanho para a pesquisa.

\section{NOTAS INFORMATIVAS}

Aprovado pela Comissão de Ética na Utilização de Animais - CEUA da UFU sob o processo número 053/11.

\section{REFERÊNCIAS}

AYRES, M.; AYRES JUNIOR, M.; AYRES, D.L. et al. BioEstat: Aplicações estatísticas nas áreas das ciências biológicas e médicas. Belém: Sociedade Civil Mamirauá, 2007. 364 p.

AZAWI, O.I. Postpartum uterine infection in cattle. Animal Reproduction Science, v.105, n.3-4, p. 187-208, 2008.

BACHA, B.; REGASSA, F.G. Subclinical endometritis in Zebu x Friesian crossbred dairy cows: its risk factors, association with subclinical mastitis and effect on reproductive performance. Tropical Animal Health and Production, v.42, n.7, p.397-403, 2010.

BAGGIOLINI, M. Chemokines and leukocyte traffic. Nature, v.392, n.6676, p.565-568, 1998.

BARLUND, C.S.; CARRUTHERS, T.D.; WALDNER, C.L. et al. A comparison of diagnostic techniques for postpartum endometritis in dairy cattle. Theriogenology, v.69, n.6, p.714-723, 2008.

BUCKLEY, F.; O'SULLIVAN, K.; MEE, J.F. et al Relationships among milk yield, body condition, cow weight, and reproduction in spring-calved Holstein-Friesians. Journal of Dairy Science, v.86, n.7, p.2308-2319, 2003.

CHEONG, S.H.; NYDAM, D.V.; GALVÃO, K.N. et al. Cow-level and herd-level risk factors for subclinical endometritis in lactating Holstein cows. Journal of Dairy Science, v.94, n.2, p.762-70, 2011. 
DUBIN, N.H.; PARMLEY, T.H.;

GHODGAONKAR, R.B. et al. Effect of

intrauterine administration of tetracycline on

cynomolgus monkeys. U.S. National Library of

Medicine National Institutes of Health, v.29,

n.6, p.561-571, 1984

DUBIN, N.H.; PARMLEY, T.H.;

GHODGAONKAR, R.B. et al. Comparative

effects of intrauterine instillation of analogues of

quinacrine and tetracycline on uterine

morphology in the rat. U.S. National Library of

Medicine National Institutes of Health, v.29,

n.6, p.553-9, 1984.

EDMONSON, A. J.; LEAN, I. J.; WEAVER, L. D. $A$ body condition scoring chat for Holstein dairy cows. Journal of Dairy Science, v.71, n.1, p.68-78, 1989.

ELLIOT, L.; MCMAHON, K.J.; GIER, H.T.; et al. Uterus of the cow after parturition: bacterial content. American Journal of Veterinary Research, v.29, n.1, p.77-81, 1968.

FEITOSA, F.L.F. Exame físico geral ou de rotina. In: Semiologia Veterinária: A Arte do Diagnóstico. 1 ed. São Paulo. Editora Roca, 2008. Cap. 4., p. 81-82.

FERREIRA, A.M. Efeito da amamentação e do nível nutricional na atividade ovariana de vacas mestiças leiteiras. 1990. Viçosa, $133 f$.

Tese (Doutorado em Zootecnia) - Curso de PósGraduação em Zootecnia, Universidade Federal de Viçosa.

GAUTAM, G.; NAKAO, T.; KOIKE, K. et al. Spontaneous recovery or persistence of postpartum endometritis and risk factors for its persistence in Holstein cows. Theriogenology, v.73, n.6, p.168-179, 2010.

GILBERT, R.O.; SCHWARK, W. S.

Pharmacologic considerations in the management of peripartum conditions in the cow. Veterinary Clinics of North America: Food Animal Practice, v.8, n.1, p.29-56, 1992.

GILBERT, R.O.; SHIN, T.S.; GUARD, C.L. et al. Prevalence of endometritis and its effects on reproductive performance of dairy cows.

Theriogenology, v.64, p. 1879-1888, 2005.

GRUNERT, E.; BIRGEL, E.H.; VALE, W.G. et al. Patologia e Clínica da Reprodução dos Animais Mamíferos- Ginecologia. Editora Livraria Varela, p. 551, 2005.

HAYIRLI, A.; GRUMMER, R. R. Factors affecting dry matter intake prepartum in relationship to etiology of peripartum lipid-related metabolic disorders: A review. Canadian
Journal of Animal Science, v.84, p. 337-347, 2004.

KASIMANICKAM, R.; DUFFIELD, T.F.; FOSTER, R.A. et al. Endometrial cytology and ultrasonography for the detection of subclinical endometritis in postpartum dairy cows.

Theriogenology, v.62, n.1, p.9-23, 2004.

KASIMANICKAM, R.; DUFFIELD, T.F.; FOSTER, R.A. et al. A comparison of the cytobrush and uterine lavage techniques to evaluate endometrial cytology in clinically normal postpartum dairy cows. Canadian Veterinary Journal, v.6, n.3, p.255-259, 2005.

KAUFMANN, T. B.; DRILLICH, M.; TENHAGEN, B. A. et al. Prevalence of bovine subclinical endometritis $4 \mathrm{~h}$ after insemination and its effects on first service conception rate. Theriogenology, v.71, n.2, p.385-391, 2009.

KÖPPEN, W. Climatologia: con un estudio de los climas de la tierra. Fondo de Cultura Econômica. México,1948. 479p.

LEBLANC, S. J.; DUFFIELD, T. F.; LESLIE, K. E.; et al. Defining and diagnosing postpartum clinical endometritis and its impact on reproductive performance in dairy cows. Journal of Dairy Science, v.85, n.9, p.2223-2236, 2002.

LEBLANC, S.J. Postpartum uterine disease and dairy herd reproductive performance: A review. The Veterinary Journal, v.176, n.1, p.102-114, 2008.

LEWIS, G.S. Uterine health and disorders. Journal of Dairy Science, v.80, n.5, p.984-994, 1997.

MARQUES JÚNIOR, A.P.; MARTINS, T.M.; BORGES, Á.M. Abordagem diagnóstica e de tratamento da infecção uterina em vacas.

Revista Brasileira Reprodução Animal, v.35, n.2, p.293-298, 2011.

MCDOUGALL, S.; HUSSEIN, H.; ABERDEIN, D. et al. Relationships between cytology, bacteriology and vaginal discharge scores and reproductive performance in dairy cattle.

Theriogenology, v.76, n.2, p.229-40, 2010.

OPSOMER, G.; GROHN, Y.T.; HERTL, J. et al. Risk factors for post partum ovarian dysfunction in high producing dairy cows in Belgium: a field study. Theriogenology, v.53, n.1, p.57, 2000.

OVERTON, T. R., WALDRON, M. R. Nutritional management of transition dairy cows: strategies to optimize metabolic health. Journal of Dairy Science, v.87, n.4, p.105-119, 2004. 
PETER, A.T.; BOSU W.T.K.; DEDECKER, R.J. Suppression of preovulatory luteinizing hormone surges in heifers after intrauterine infusions of Escherichia coli endotoxin. American Journal of Veterinary Research, v.50, n.1, p.73, 1989.

PLÖNTZKE, J.; MADOZ, L.V.; DE LA SOTA, R.L. et al. Subclinical endometritis and its impact on reproductive performance in grazing dairy cattle in Argentina Animal Reproduction Science, v.122, n.1-2, p.52-57, 2010.

PLÖNTZKE J.; MADOZ, L.V.; DE LA SOTA, R.L. et al. Prevalence of Clinical Endometritis and its Impact on Reproductive Performance in Grazing Dairy Cattle in Argentina. Reproduction in Domestic Animals, v.46, n.3, p.520-526, 2011.

ROCHE, J. F. The effect of nutritional management of the dairy cow on reproductive efficiency. Animal Reproduction Science, v.96, n.3-4, p.282-296, 2006.

SALASEL, B.; MOKHTARI, A.; TAKTAZ, T. Prevalence, risk factors for and impact of subclinical endometritis in repeat breeder dairy cows. Theriogenology, v.74, n.7, p.1271-1278, 2010.

SANTOS, T.M.; CAIXETA, L.S.; MACHADO, V.S. et al. Antimicrobial resistance and presence of virulence factor genes in Arcanobacterium pyogenes isolated from the uterus of postpartum dairy cows. Veterinary Microbiology. v.28, p. 84-89, 2010.

SAUT, J.P.E.; OLIVEIRA R. S. B. R.; MARTINS, C. F. G. et al. The clinical evaluation of physiological postpartum uterine involution in mixed-breed dairy cows. In: XXVI WORLD BUIATRICS CONGRESS 2010, 26. 2010, Santiago, Proceedings... Santiago, Chile.

SHAMS-ESFANDABADI, N.; SHIRAZI, H.; GHASEMZADEH-NAVA, H. Pregnancy rate following post-insemination intrauterine treatment of endometritis in dairy cattle. Journal Veterinary Medicine, v.51, p.155-156, 2004.

SHELDON, I.M.; NOAKES D.E.; RYCROFT, A.N.; et al. Influence of uterine bacterial contamination after parturition on ovarian dominant follicle selection and follicle growth and function in cattle. Reproduction, v.123, p.837845. 2002.

SHELDON, I. M. The Postpartum uterus. Veterinary Clinics Food Animal, v.20, n.3, p.569-591, 2004.

SHELDON, I.M.; DOBSON, H. Postpartum uterine health in cattle. Animal Reproduction Science, v.82-83, p.295-306, 2004.
SHELDON, I.M.; LEWIS, G.S.; LEBLANC, S.; et al. Defining postpartum uterine disease in cattle. Theriogenology, v.65, n.8, p.1516-1530, 2006.

SHELDON, I.M. Endometritis in cattle: pathogenesis, consequences for fertility, diagnosis and therapeutic recommendations. Partners in Reproduction, v.2, n.1, p.1-5, 2007.

SHELDON, I.M.; PRICE, S.B.; CRONIN, J. et al. Mechanisms of infertility associated with clinical and subclinical endometritis in high producing dairy cattle. Reproduction in Domestic Animals, v.44, n.1, p.1-9, 2009

SMITH, B.I.; RISCO, C.A. Management of periparturient disorders in dairy cattle. Veterinary Clinics: Food Animal Practice, v. 21, p.503-52, 2005.

WILLIAMS, E. J.; FISHER, D. P.; PFEIFFER, D. $U$.; et al. Clinical evaluation of postpartum vaginal mucus reflects uterine bacterial infection and the immune response in cattle.

Theriogenology, v.63, n.1, p.102-117, 2005.

VALLADA, E. P. Atheneu, Manual de Técnicas Hematológicas, São Paulo: 1999.

VIEIRA, S. Bioestatística -Tópicos

Avançados. 2.ed. Rio de Janeiro. Editora Elsevier, 2003.

ZANEVELD, L.J.D.; GOLDSMITH, A. Lack of tubal occlusion by intrauterine quinacrine and tetracycline in the primate, Contraception, v.30, n. 2, p.153-9, 1984. 